\title{
GENERALIZED SEMIMAGIC SQUARES FOR DIGITAL HALFTONING
}

\author{
AKITOSHI KAWAMURA
}

\begin{abstract}
Completing Aronov et al.'s study on zero-discrepancy matrices for digital halftoning, we determine all $(m, n, k, l)$ for which it is possible to put $m n$ consecutive integers on an $m \times n$ board (with wrap-around) so that each $k \times l$ region has the same sum. For one of the cases where this is impossible, we give a heuristic method to find a matrix with small discrepancy.
\end{abstract}

A semimagic square is a square matrix whose entries are consecutive integers and which has equal row and column sums. One way to generalize this millennia-old concept is to specify the sums on regions other than rows and columns. Ingenious constructions of squares satisfying various sum constraints have been described by many professional and amateur mathematicians. While most of them are interested in adding more and more constraints to make their squares impressive, one can generally consider sum conditions on any set of regions.

Aronov et al. [1] took up this problem for square regions: is there an $n \times n$ matrix with entries $0, \ldots, n^{2}-1$ such that every $k \times k$ region has the same sum? It is amusing to note that this variant of the classical problem is motivated by an engineering question of finding good dither matrices for digital halftoning, a method to approximate a continuous-tone image by a binary image for printing (see their paper for details). They showed [1, Theorem 1], using what they call constant-gap matrices, that the answer is yes if $k$ and $n$ are even or if $n$ is an integer power of $k$, and no if $k$ and $n$ are relatively prime or if $k$ is odd and $n$ is even. We will solve this problem completely by determining all $(n, k)$ for which such matrices exist (Section 1$)$. Our construction of the matrices is much simpler even for the cases that have already been settled positively. We also give counterexamples to Asano et al.'s conjecture on the smallest possible discrepancy when $n$ is odd and $k=2$ (Section 2).

Definitions. For a positive integer $N$, we write $[N]=\{0,1, \ldots, N-1\}$. The remainder when an integer $x$ is divided by $N$ belongs to $[N]$ and is denoted by $x \bmod N$.

We consider the slightly generalized setting where the matrices and regions are rectangles instead of squares. Let $m$ and $n$ be positive integers. For an $m \times n$ matrix $D$ and index $(i, j) \in[m] \times[n]$, we denote the $(i, j)$ th entry of $D$ by $D(i, j)$. Any set $R \subseteq[m] \times[n]$ of indices is called a region. The sum of the numbers on $R$ is denoted by $D(R)=\sum_{(i, j) \in R} D(i, j)$. The discrepancy of $D$ with respect to a set $\mathscr{R}$ of regions is the difference between the maximum and minimum $D(R)$ as $R$ varies in $\mathscr{R}$. When it is zero, $D$ is said to be $\mathscr{R}$-uniform.

The translate of $R$ by $(a, b) \in \mathbf{Z}^{2}$ is denoted by

$$
R+(a, b)=\{((i+a) \bmod m,(j+b) \bmod n):(i, j) \in R\} \subseteq[m] \times[n] .
$$

The set of all translates of $R$ is denoted by $\bar{R}=\left\{R+(a, b):(a, b) \in \mathbf{Z}^{2}\right\}$. 


\begin{tabular}{|c|c|c|c|c|}
\hline 14 & 1 & 21 & 0 & 18 \\
\hline 16 & 13 & 9 & 22 & 4 \\
\hline 5 & 17 & 12 & 7 & 19 \\
\hline 20 & 2 & 15 & 11 & 8 \\
\hline 6 & 24 & 3 & 23 & 10 \\
\hline
\end{tabular}

Figure 1 . This $5 \times 5$ table $D$ has discrepancy 8 with respect to $\overline{[2] \times[2]}$, because $44 \leq D(R) \leq 52$ for every $2 \times 2$ region $R$.

By an $m \times n$ table we mean an $m \times n$ matrix in which each element of $[m n]$ appears exactly once. We are interested in tables with small (or zero) discrepancy with respect to $\overline{[k] \times[l]}$, the set of all $k$-by-l rectangles (Figure 1 ).

\section{ZERO DISCREPANCY}

The greatest common divisor of positive integers $x$ and $y$ is denoted by $\operatorname{gcd}(x, y)$. The goal of this section is to show the following:

Theorem 1. Let $m, n, k, l$ be positive integers with $k<m$ and $l<n$. Let $k^{\prime}=$ $\operatorname{gcd}(k, m)$ and $l^{\prime}=\operatorname{gcd}(l, n)$. Then there exists a $\overline{[k] \times[l]}$-uniform $m \times n$ table if and only if $k^{\prime}$ and $l^{\prime}$ are greater than 1 and $k^{\prime} l^{\prime}(m n-1)$ is even.

This is an immediate consequence of the following Lemmas 2 and 3.

Lemma 2. $A \overline{[k] \times[l]}$-uniform $m \times n$ matrix is $\overline{[\operatorname{gcd}(k, m)] \times[\operatorname{gcd}(l, n)]}$-uniform.

Proof. Let $D$ be a $\overline{[k] \times[l]}$-uniform $m \times n$ matrix. We will show that $D$ is $\overline{\left[k^{\prime}\right] \times[l]}$ uniform, where $k^{\prime}=\operatorname{gcd}(k, m)$. We get the conclusion of the lemma by repeating the same argument with rows and columns switched.

For each $(i, j) \in[m] \times[n]$, the regions $\left[k^{\prime}\right] \times[l]+(i, j)$ and $\left[k^{\prime}\right] \times[l]+(i+k, j)$ have the same sum on $D$, because each of them combined with $\left[k-k^{\prime}\right] \times[l]+\left(i+k^{\prime}, j\right)$ makes a $k \times l$ rectangle. Thus for each $(i, j) \in[m] \times[n]$, the rectangles

$$
\left[k^{\prime}\right] \times[l]+(i+q k, j), \quad q \in\left[m / k^{\prime}\right],
$$

all have the same sum on $D$. Since $k^{\prime}=\operatorname{gcd}(k, m)$, these $m / k^{\prime}$ rectangles cover the strip $[m] \times[l]+(0, j)$ without overlap. Hence,

$$
\begin{aligned}
\frac{m}{k^{\prime}} \cdot D\left(\left[k^{\prime}\right] \times[l]+(i, j)\right) & =\sum_{q \in\left[m / k^{\prime}\right]} D\left(\left[k^{\prime}\right] \times[l]+(i+q k, j)\right) \\
& =D([m] \times[l]+(0, j))=\frac{1}{k} \sum_{r \in[m]} D([k] \times[l]+(r, j)) .
\end{aligned}
$$

Since the rightmost side is a constant independent of $(i, j)$ by $\overline{[k] \times[l]}$-uniformity, so is the leftmost side. Thus $D$ is $\overline{\left[k^{\prime}\right] \times[l]}$-uniform.

Lemma 3. Let $m$ and $n$ be positive integers, and let $k<m$ and $l<n$ be their positive

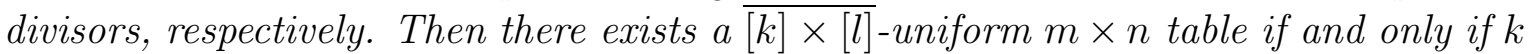
and $l$ are greater than 1 and $k l(m n-1)$ is even. 


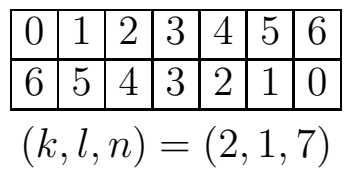

\begin{tabular}{c|l|l|l|l|l|l|l|}
\hline 0 & 1 & 2 & 3 & 4 & 5 & 6 \\
\hline 3 & 4 & 5 & 6 & 0 & 1 & 2 \\
\hline 6 & 4 & 2 & 0 & 5 & 3 & 1 \\
\hline$(k, l, n)=(3,1,7)$
\end{tabular}

\begin{tabular}{|l|l|l|l|l|l|l|l|}
\hline 0 & 4 & 1 & 5 & 2 & 6 & 3 & 7 \\
\hline 0 & 4 & 1 & 5 & 2 & 6 & 3 & 7 \\
\hline 7 & 6 & 5 & 4 & 3 & 2 & 1 & 0 \\
$c$ & $(k, l, n=(3,2,8)$
\end{tabular}

Figure 2. Examples of matrices of Lemma 4.

One direction is a simple generalization of $[1$, Theorem $1(\mathrm{~b}, \mathrm{c})]$ :

Proof of the "only if" part of Lemma 3. Let $D$ be a $\overline{[k] \times[l]}$-uniform $m \times n$ table. It is easy to see that $D(R)=k l(m n-1) / 2$ for each $R \in \overline{[k] \times[l]}$. Since $D(R)$ must be an integer, the second claim follows. For the first claim, assume $k=1$ for contradiction (the case $l=1$ is similar). Then $D([1] \times[l])=D([1] \times[l]+(0,1))$ and hence $D(0,0)=$ $D(0, l)$, contradicting the assumption that $D$ is a table.

For the converse, we use the building blocks provided by the following lemma:

Lemma 4. Let $k>1$ and $l>0$ be integers and let $n$ be a positive multiple of $l$. If $k l(n-1)$ is even, then there exists a $\overline{[k] \times[l]}$-uniform $k \times n$ matrix in which each row is a permutation of $[n]$.

Proof. A $\overline{[k] \times[l]}$-uniform $k \times n$ matrix and a $\overline{\left[k^{\prime}\right] \times[l]}$-uniform $k^{\prime} \times n$ matrix stacked vertically make a $\overline{\left[k+k^{\prime}\right] \times[l]}$-uniform $\left(k+k^{\prime}\right) \times n$ matrix. Also, a $\overline{[k] \times[l]}$-uniform matrix is $\overline{[k] \times\left[l^{\prime}\right]}$-uniform for any multiple $l^{\prime}$ of $l$. Therefore, it suffices to construct the desired matrix $P$ for the cases $(k, l)=(2,1),(3,1)$ and $(3,2)$ (Figure 2). If $(k, l)=(2,1)$, let

$$
P(0, j)=j, \quad P(1, j)=n-1-j .
$$

If $(k, l)=(3,1)$, then $n$ is odd by the assumption; let

$$
P(0, j)=j, \quad P(1, j)=\left(j+\frac{n-1}{2}\right) \bmod n, \quad P(2, j)=(-2 j-1) \bmod n .
$$

If $(k, l)=(3,2)$, let

$$
P(0, j)=P(1, j)=\left\lfloor\frac{j}{2}\right\rfloor+\frac{n}{2}(j \bmod 2), \quad P(2, j)=n-1-j .
$$

It is easy to verify that $P$ is $\overline{[k] \times[l]}$-uniform in each case.

Proof of the "if" part of Lemma 3. We may assume without loss of generality that $l(m n-1)$ is even. In this case, both $k l(n-1)$ and $l(m / k-1)$ are even, so by Lemma 4 , there are a $\overline{[k] \times[l]}$-uniform $k \times n$ matrix $P$ whose rows are permutations of $[n]$, and a $\overline{[l] \times[1]}$-uniform $l \times(\mathrm{m} / \mathrm{k})$ matrix $Q$ whose rows are permutations of $[\mathrm{m} / \mathrm{k}]$. Define an $m \times l$ matrix $T$ by

$$
T(a, j)=Q(j,\lfloor a / k\rfloor) k+(a \bmod k) .
$$

Then $T$ is $\overline{[k] \times[l]}$-uniform and its columns are permutations of $[m]$. Define an $m \times n$ matrix $D$ by

$$
D(a, b)=P(a \bmod k, b) m+T(a, b \bmod l)
$$




$$
\begin{aligned}
& D=\left[\begin{array}{l}
P \\
P \\
P
\end{array}\right] \times 9+\left[\begin{array}{llll}
T & T & T & T
\end{array}\right] \\
& \begin{array}{|l|l|l|l|l|l|l|l|}
\hline 0 & 4 & 1 & 5 & 2 & 6 & 3 & 7 \\
\hline 0 & 4 & 1 & 5 & 2 & 6 & 3 & 7 \\
\hline 7 & 6 & 5 & 4 & 3 & 2 & 1 & 0 \\
\hline 0 & 4 & 1 & 5 & 2 & 6 & 3 & 7 \\
\hline 0 & 4 & 1 & 5 & 2 & 6 & 3 & 7 \\
\hline 7 & 6 & 5 & 4 & 3 & 2 & 1 & 0 \\
\hline 0 & 4 & 1 & 5 & 2 & 6 & 3 & 7 \\
\hline 0 & 4 & 1 & 5 & 2 & 6 & 3 & 7 \\
\hline 7 & 6 & 5 & 4 & 3 & 2 & 1 & 0 \\
\hline
\end{array} \times 9+\begin{array}{|l|l|l|l|l|l|l|l|}
\hline 0 & 6 & 0 & 6 & 0 & 6 & 0 & 6 \\
\hline 1 & 7 & 1 & 7 & 1 & 7 & 1 & 7 \\
\hline 2 & 8 & 2 & 8 & 2 & 8 & 2 & 8 \\
\hline 3 & 3 & 3 & 3 & 3 & 3 & 3 & 3 \\
\hline 4 & 4 & 4 & 4 & 4 & 4 & 4 & 4 \\
\hline 5 & 5 & 5 & 5 & 5 & 5 & 5 & 5 \\
\hline 6 & 0 & 6 & 0 & 6 & 0 & 6 & 0 \\
\hline 7 & 1 & 7 & 1 & 7 & 1 & 7 & 1 \\
\hline 8 & 2 & 8 & 2 & 8 & 2 & 8 & 2 \\
\hline 4
\end{array}=\begin{array}{|l|l|l|l|l|l|l|l|}
\hline 0 & 42 & 9 & 51 & 18 & 60 & 27 & 69 \\
\hline 1 & 43 & 10 & 52 & 19 & 61 & 28 & 70 \\
\hline 65 & 62 & 47 & 44 & 29 & 26 & 11 & 8 \\
\hline 4 & 40 & 13 & 49 & 22 & 58 & 31 & 67 \\
\hline 68 & 59 & 50 & 41 & 32 & 23 & 14 & 5 \\
\hline 6 & 36 & 15 & 45 & 24 & 54 & 33 & 63 \\
\hline 7 & 37 & 16 & 46 & 25 & 55 & 34 & 64 \\
\hline 71 & 56 & 53 & 38 & 35 & 20 & 17 & 2 \\
\hline
\end{array}
\end{aligned}
$$

Figure 3. Construction of $D$ for $(k, l, m, n)=(3,2,9,8)$.

(Figure 3). Since $P$ and $T$ are $\overline{[k] \times[l]}$-uniform, so is $D$. To see that $D$ is a table, suppose that $D(a, b)=D\left(a^{\prime}, b^{\prime}\right)$. By (7) and (8) we see that

$$
\left\{\begin{aligned}
P(a \bmod k, b) & =P\left(a^{\prime} \bmod k, b^{\prime}\right), \\
Q(b \bmod l,\lfloor a / k\rfloor) & =Q\left(b^{\prime} \bmod l,\left\lfloor a^{\prime} / k\right\rfloor\right), \\
a \bmod k & =a^{\prime} \bmod k .
\end{aligned}\right.
$$

Since $P$ 's rows are permutations, the first and the third equation imply that $b=b^{\prime}$. Since $Q$ 's rows are permutations, this and the second equation imply that $a=a^{\prime}$.

In the above, we constructed the uniform table as a linear combination of two uniform matrices with smaller entries. This idea is due to Euler [3] who gave a construction of a semimagic square (that is, a $(\overline{[1] \times[n]} \cup \overline{[n] \times[1]}$ )-uniform $n \times n$ table) from a pair of special $(\overline{[1] \times[n]} \cup \overline{[n] \times[1]})$-uniform matrices called Latin squares.

\section{FINDING LOW-DISCREPANCY TABLES BY RANKING}

In this section, we confine ourselves, as Asano et al. [2] did, to the case where $k=l=2$ and $m=n$. Theorem 1 states that in this case a uniform table exists if and only if $n$ is even. For odd $n$ 's, they construct a table with discrepancy $2 n$, and conjecture that it is the smallest possible. This is refuted by our Figures 1 and 4 . Figure 1 was discovered by an exhaustive search. We describe briefly how Figure 4 was obtained.

Define $f:[0,1]^{2} \rightarrow \mathbf{R}$ by $f(x, y)=g(x)+g(y)$, where

$$
g(x)= \begin{cases}1-(4 x-1)^{2} & \text { if } x \leq 1 / 2 \\ -1+(4 x-3)^{2} & \text { if } x \geq 1 / 2\end{cases}
$$

(Figure 5). Let $\alpha, \beta \in[0,1]$ and define $s:[n]^{2} \rightarrow[0,1]^{2}$ by

$$
s(i, j)=\left(\frac{i+\alpha}{n}, \frac{j+\beta}{n}\right) .
$$


433523439519445511453507460497465490472486478480482476487470492462500458508450515442520437525 616348603362592378576393556411543427524449494479471505441529422547403564387581374594357607345 283675298655312634337614356590383560416531451483498436538405570377595350622327643306662292678 727241716253692278669305637340604375569418522475454535401580361615329646300677273701249721238 198762206747225720255686293649328608376559428488517413578358625320660279695248729218751201765 797166786180769207733245698282652330602385539469434558371621315670270706233746199775173790163 132823141813160782196745237703284648342588414491530388606324668269714223755185792156818137827 856109848120826150793191749239696294635359553464425583341653275712221764174802140831116852107 828768886211383314579619274424768430761039749654137063329170422676117281013184310286784879 $898 \quad 61893 \quad 7886910383614779119773225866733957246141759831068524275217780813085194880 \quad 73895 \quad 58$ $4391650900 \quad 7187210483215278121071828063238150455034765825973619379813684991884 \quad 65906 \quad 48919$ $933 \quad 30924 \quad 46903 \quad 7286811482416176822968831758645640262329071520978314883910088359912 \quad 37928 \quad 28$ $1494125927 \quad 47899 \quad 79861123811181742256651369509561331682234766162825111871689113693420943$ $9531094426923 \quad 51892 \quad 89846144784208713299597448394638267734190804126859 \quad 80901 \quad 42930 \quad 18950 \quad 8$

$2955 \quad 11940 \quad 31915 \quad 64873112821169757244671354514571314699215778153838 \quad 95889 \quad 54921 \quad 24946 \quad 6958$ $960 \quad 3952 \quad 17932 \quad 44896 \quad 83853134794200725289609444384647257743179814117866 \quad 70909 \quad 34939 \quad 12956 \quad 0$

$1957 \quad 9942 \quad 29917 \quad 60875110822167759243673351516574311702214779149840 \quad 93890 \quad 52925 \quad 22949 \quad 4959$ $954 \quad 794721926 \quad 49894 \quad 87850139788205717297599446389642264737186809124863 \quad 76904 \quad 39936 \quad 15951 \quad 5$ $13945199314190575864118815175748251656366510563326687228770158829105878 \quad 6291432937 \quad 16948$ $938 \quad 27929 \quad 38910 \quad 67874106830157774222694309591455400626285722203789142845 \quad 90887 \quad 56918 \quad 33935 \quad 23$ $3592045907 \quad 66882 \quad 99841146787202726276639379506551344666252741184806128855 \quad 85891 \quad 57913 \quad 40922$ $908 \quad 55897 \quad 6988197847135799189739250672334575459415605304691232760170817121857 \quad 86886 \quad 63902 \quad 53$ $748858187010184413380517875323669030161839249954436564128171021677216481912285492877 \quad 77888$ 8659885811583413880117675622770528764435555746342358733566126572421277316581612984210886096 119835127820151795183754224711274657333593410493532382613313676260728213771171803143828125837 812155800168780195750231707272665323611380545468432566360628302679261723219758187785159807154 182776194763211731246700277664321620367567424489521406584349630303674268708235740204767188777 738220730240709263681295650325619364577408527474447542395589352627316659286689254719230735217 262693271680296654318629343601372573404536443485503430548396582363612336640308663288683266697 645322631338617353596373579391554419533438502477466513431540409562386585368600346624332636319 390565399552412546421534429526440512457495473481484467501452518435528426537420549407555398568

Figure 4 . A $31 \times 31$ table whose discrepancy with respect to $\overline{[2] \times[2]}$ is 27 .

Let $H$ be the $n \times n$ table whose $(i, j)$ th entry is the rank of $f(s(i, j))$ (with some tie-breaking rule):

$$
\begin{aligned}
H(i, j)=\mid\left\{\left(i^{\prime}, j^{\prime}\right) \in[n]^{2}:\right. & f\left(s\left(i^{\prime}, j^{\prime}\right)\right)<f(s(i, j)) \text { or } \\
& \left.\left(f\left(s\left(i^{\prime}, j^{\prime}\right)\right)=f(s(i, j)) \text { and } n i^{\prime}+j^{\prime}<n i+j\right)\right\} \mid .
\end{aligned}
$$

Finally, define the desired matrix $D$ by

$$
D((i+j) \bmod n,(i-j) \bmod n)=H(i, j) .
$$

Figure 4 was obtained by this method with $n=31$ and $(\alpha, \beta)=(0.286,0)$.

To see intuitively why $D$ has small discrepancy, note that a $2 \times 2$ region in $D$ corresponds to the region in $H$ (or its translate) shown in Figure 6 . These four cells are mapped by $s$ to two nearby points $(x \pm \varepsilon, y)$ and another two points $(x+1 / 2, y+1 / 2 \pm \varepsilon)$ (the coordinates are modulo 1$)$. Since $f(x, y)=-f(x+1 / 2, y+1 / 2)$, the sum of the values of $f$ at these four points is almost zero. Thus, assuming that taking the ranks does not distort the distribution of values too much, we can expect that $D$ has low discrepancy. We add the displacement $(\alpha, \beta)$ in $(11)$ in order to reduce the chance of ties in the ranking which seem to work adversely.

As Aronov et al. [1] point out, our problem is analogous to a common situation in discrete geometry where we try to arrange discrete objects so that they look close to some "balanced" continuous distribution. The constraint peculiar to our problem is 

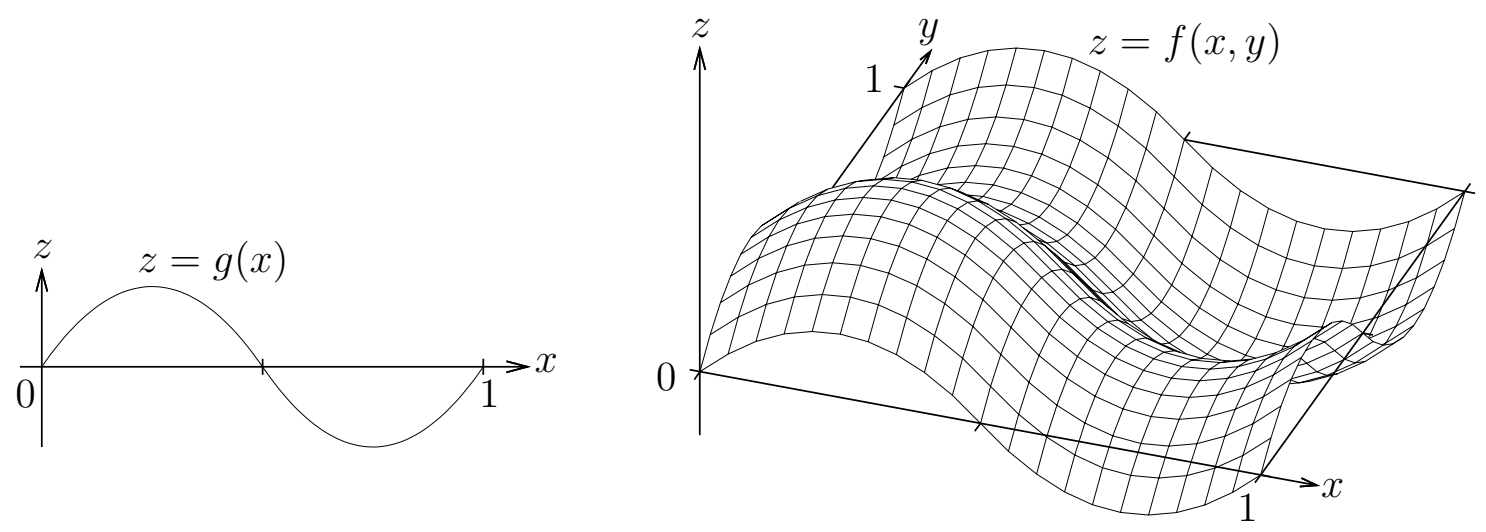

Figure 5. The functions $g$ and $f$.

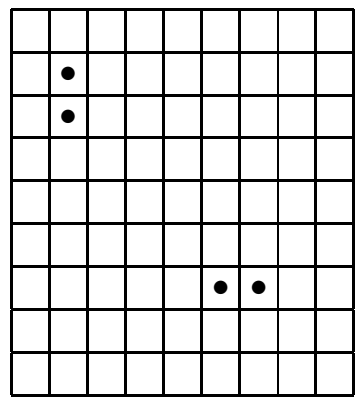

Figure 6 . A region in $H$ corresponding to a $2 \times 2$ square in $D($ for $n=9)$.

that we have to use each number in $[m n]$ exactly once. The ranking technique used here may be applicable to other problems with this constraint. However, analyzing its performance seems to be hard: although our computer experiment for several $n$ 's suggests that the above method achieves sublinear $2 \times 2$ discrepancy, we have no proof yet.

Acknowledgments. The author thanks Tomoko Adachi, Tetsuo Asano, Tsukasa Kuribayashi, Yasuko Matsui, Shao-Chin Sung, Hideki Tsuiki, Ryuhei Uehara and the referees for helpful comments and discussions. This work was supported in part by the Nakajima Foundation and the Natural Sciences and Engineering Research Council of Canada. An earlier version was presented at the Eighth Japan-Korea Joint Workshop on Algorithms and Computation (WAAC 2005).

\section{REFERENCES}

[1] B. Aronov, T. Asano, Y. Kikuchi, S. C. Nandy, S. Sasahara, and T. Uno. A generalization of magic squares with applications to digital halftoning. Theory of Computing Systems 42(2), pp. 143-156, 2008.

[2] T. Asano, S. Choe, S. Hashima, Y. Kikuchi, and S.-C. Sung. Distributing distinct integers uniformly over a square matrix with application to digital halftoning. Information Processing Society of Japan (IPSJ) SIG Technical Report AL-100, 2005(26), pp. 79-86, 2005.

[3] L. Euler. De quadratis magicis. Commentationes arithmeticae 2, pp. 593-602, 1849. Presented to the St. Petersburg Academy in 1776. 